Faculty of Mathematical Sciences

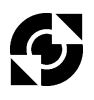

University of Twente

The Netherlands
P.O. Box 217

7500 AE Enschede

The Netherlands

Phone: +31-53-4893400

Fax: +31-53-4893114

Email: memo@math.utwente.nl

www.math.utwente. $\mathrm{nl} /$ publications

Memorandum No. 1665

Integrable hierarchies and

the WDVV-equations

G.F. HELMINCK

December, 2002

ISSN 0169-2690 


\title{
INTEGRABLE HIERARCHIES AND THE WDVV-EQUATIONS
}

\author{
G.F. HELMINCK
}

\begin{abstract}
In this paper an overview is given of connections between the Witten-Dijkgraaf-VerlindeVerlinde equations and its generalization and various integrable systems. In particular its link with the $n$-component $K P$-hierarchy is treated in a fairly detailed way.
\end{abstract}

\section{INTRODUCTION}

There are many instances where soliton equations of $K d V$-type play a role in quantum field theory, one of the most prominent ones being the Witten conjectures that relate partition functions of topological field theories to $\tau$-functions of integrable hierarchies. Here we give an overview of connections between the Witten- Dijkgraaf-Verlinde-Verlinde-equations from 2-dimensional topological $N=2 \mathrm{su}-$ perconformal field theory and various integrable hierarchies, more in particular with the $n$-component $K P$-hierarchy.

The contents of the remaining sections is as follows. The second section gives a quick introduction to the WDVV-equations and their generalization that shows up in Seiberg-Witten theory. The first system can be given a coordinate-free description as a Frobenius manifold, of which we recall the essentials in the first part of the third section. Next we limit our attention to a subclass, the semisimple Frobenius manifolds. This leads naturally to the Darboux-Egoroff system and its linearization. In the fourth section we point out a number of links that have been laid between integrable hierarchies and the WDVV-equations and vice-versa. Since we intend to elaborate here on the connection with the $n$-component $K P$-hierarchy, the next section is devoted to a crash course on the essentials of this integrable system. It starts with a description of the equations and their linearization. Next it is shown that in order to obtain solutions of the hierarchy it suffices to construct so-called wavefunctions. A useful characterization of these wavefunctions is by means of the bilinear relations and a pure algebraic setting for them is presented. The section concludes with the Grassmannian construction of wavefunctions. The last section shows how one can obtain within the Sato Grassmannian the different relations in the Darboux-Egoroff system and how to construct from the wavefunction a solution of the linearization of the Darboux-Egoroff system.

\section{The WDVV-EQuations and its generalization}

To give a direct idea of the form of the equations in question, we present here its local form that allows already interesting generalizations. The whole question concerns a holomorphic function $F\left(x_{1}, \ldots, x_{n}\right)$ in $n$ complex variables. For each $i, 1 \leq i \leq n$, we denote by $F(i)$ the Hessian matrix of $\frac{\partial F}{\partial x_{i}}$, i.e.

$$
F(i)_{j k}=\frac{\partial^{3} F}{\partial x_{i} \partial x_{j} \partial x_{k}} .
$$

Now we have the following

Definition 2.1. We call $F$ a solution of the WDVV-equations or Associativity equations if the following relations hold for $F$ :

(1) The matrix $\mathrm{F}(1)$ is constant and invertible.

1991 Mathematics Subject Classification. 53D45, 22E65, 14M15, 35Q58,81T45.

Key words and phrases. Frobenius manifolds, Witten-Dijkgraaf-Verlinde-Verlinde-equations, Integrable hierarchies. 
(2) For all $i, 1 \leq i \leq n$, and $j, 1 \leq j \leq n, F(i) F(1)^{-1} F(j)=F(j) F(1)^{-1} F(i)$.

Remark 2.2. Witten, Dijkgraaf, Verlinde and Verlinde obtained matrices satisfying these equations as the three point correlators of the primary fields of a perturbed two dimensional topological field theory. The function $F$ corresponds then to the primary free energy of the model.

To explain the second name in the definition, let $C(i):=F(1)^{-1} F(i), 1 \leq i \leq n$. We can use these matrices to put a product structure on the tangent space in a point by putting

$$
\frac{\partial}{\partial x_{i}} \circ \frac{\partial}{\partial x_{k}}:=\sum_{j=1}^{n} C(i)_{j k} \frac{\partial}{\partial x_{j}} .
$$

One verifies directly that this product structure is commutative and associative thanks to the second property, which can also be written as $C(i) C(j)=C(j) C(i)$ for all $i$ and $j$. Clearly, differentiation w.r.t. $x_{1}$ is the unit element for this product structure.

Remark 2.3. For the solutions of the WDVV-equations that relate to topological field theory, one requires still invariance w.r.t. a number of scaling transformations and this results in the condition: the function $F$ is quasi-homogeneous, i.e.

$$
F\left(\lambda^{d_{1}} x_{1}, \ldots, \lambda^{d_{r}} x_{n}\right)=\lambda^{d_{F}} F\left(x_{1}, \ldots, x_{n}\right),
$$

for certain numbers $d_{F}, d_{1}, \ldots, d_{n}$. In this paper we restrict our attention to the 2 conditions in the definition 2.1 and leave this third condition out of consideration.

If there is not a privileged direction like $x_{1}$, then the system of equations in 2.1 can be generalized as follows

Definition 2.4. We call $F$ a solution of the generalized WDVV-equations if the following relation hold for $F$ : there is an invertible matrix $G=\sum_{i=1}^{n} a_{i} F(i)$ such that for all $i, 1 \leq i \leq n$, and $j, 1 \leq j \leq n$,

$$
F(i) G^{-1} F(j)=F(j) G^{-1} F(i)
$$

Also this system of equations corresponds to an associativity condition: simply replace $F(1)$ by $G$ in the definition of $C(i)$ above. Remarkably, it possesses also solutions coming from models in physics. It was shown by Marshakov, Mironov and Morozov that the full prepotential for $N=2$ Super-Yang-Mills theory for the simple classical Lie algebras satisfies this generalized WDVV-system, see [MMM97]. By a different method Ito and Yang, see [IY98], showed this to be true also for the groups of type $E$ and the last nontrivial remaining case, that of $F_{4}$, was solved in [HKM01].

\section{Geometric Description}

3.1. Frobenius manifolds. To globalize the structure occurring in the first section, one considers a complex manifold $M$ with its structure sheaf $\mathcal{O}_{M}$. The tangent sheaf on $M$ we denote by $\mathcal{T}_{M}$ and the cotangent sheaf by $\mathcal{T}_{M}^{*}$. The first piece of structure we need is

Definition 3.2. An affine flat structure on $M$ is a subsheaf $\mathcal{T}_{M}^{f}$ of linear spaces of pairwise commuting vectorfields such that $\mathcal{T}_{M}=\mathcal{O}_{M} \otimes_{\mathbb{C}} \mathcal{T}_{M}^{f}$. The sections of $\mathcal{T}_{M}^{f}$ are called flat vector fields.

Locally, one can find coordinates $x_{1}, \ldots, x_{n}$ on $M$, the so-called flat coordinates, such that the $\left\{\tilde{\partial}_{i}:=\right.$ $\left.\frac{\partial}{\partial x_{i}}\right\}$ form a basis of $\mathcal{T}_{M}^{f}$.

The second ingredient is a metric on $M$, i.e. a symmetric pairing $g: S^{2}\left(\mathcal{T}_{M}\right) \rightarrow \mathcal{O}_{M}$. This induces an isomorphism $\tilde{g}: \mathcal{T}_{M} \rightarrow \mathcal{T}_{M}^{*}$. We want the metric $g$ to be compatible with the affine structure $\mathcal{T}_{M}^{f}$, i.e. $g(X, Y)$ is constant for flat vector fields $X$ and $Y$. The constant matrix $\left\{g_{a b}:=g\left(\tilde{\partial}_{a}, \tilde{\partial}_{b}\right)\right\}$ corresponds then with the matrix $F(1)$ in the privileged direction.

To get to the multiplication on the tangent spaces, one needs a symmetric tensor $A: S^{3}\left(\mathcal{T}_{M}\right) \rightarrow \mathcal{O}_{M}$. This leads to the following implicit definition of the multiplication $\circ$ on $\mathcal{T}_{M}$ :

$$
g(X \circ Y, Z):=A(X, Y, Z)
$$


for all vector fields $X, Y$ and $Z$. Note that this definition implies that the metric is invariant w.r.t. this multiplication, i.e.

$$
g(X \circ Y, Z)=g(X, Y \circ Z) .
$$

If we write $A_{a b c}:=A\left(\tilde{\partial}_{a}, \tilde{\partial}_{b}, \tilde{\partial}_{c}\right)$ and $\left(g^{a b}\right)$ for the inverse of the matrix $\left(g_{a b}\right)$, then this multiplication is locally given by

$$
\tilde{\partial}_{a} \circ \tilde{\partial}_{b}=\sum_{c=1}^{n}\left\{\sum_{e=1}^{n} A_{a b e} g^{e c}\right\} \tilde{\partial}_{c} .
$$

Since $A$ is symmetric this multiplication o is commutative, but the associativity does not have to hold. Also there does not have to be, locally, a function $F$ such that

$$
A_{a b c}=\frac{\partial^{3} F}{\partial x_{a} \partial x_{b} \partial x_{c}} .
$$

By adding these two properties to the structures just introduced, one arrives at the global object corresponding to the WDVV-equations

Definition 3.3. Let $M$ be a complex manifold. We say that the triple $\left(\mathcal{T}_{M}^{f}, g, A\right)$ makes $M$ into a Frobenius manifold, if the multiplication "o" is associative and the tensor $A$ is locally potential, i.e. for all flat vector fields $X, Y$ and $Z$

$$
A(X, Y, Z)=(X Y Z) F
$$

for some holomorphic function $F$.

Remark 3.4. The structure of Frobenius manifolds was treated extensively in the lecture notes [Du96]. The present short presentation of Frobenius manifolds is based on [Manin99]. There one can also find a description of the role they play in quantum cohomology and at Gromov-Witten invariants. The proper globalization of the generalized $W D V V$-equations seems to be open still.

Dubrovin showed that Frobenius manifolds form the moduli space of 2-dimensional topological field theories. As such any link to these models means a connection with the WDVV-equations.

3.5. Semisimplicity. An important class of Frobenius manifolds are the following ones

Definition 3.6. Let $\left(M, \mathcal{T}_{M}^{f}, g, A\right)$ be a Frobenius manifold. It is called semi-simple, if there exists locally a basis $X_{1}, \ldots, X_{r}$ of $\mathcal{T}_{M}$ such that the multiplication on $\mathcal{T}_{M}$ is given by

$$
\sum_{i=1}^{n} f_{i} X_{i} \circ \sum_{i=1}^{n} g_{i} X_{i}=\sum_{i=1}^{n} f_{i} g_{i} X_{i}
$$

In other words the tangent spaces at every point of $M$ are semi-simple algebras. Dubrovin gave in [Du96] a complete description of this class and the link with the Darboux-Egoroff system. We resume the results from him that we need in a

Theorem 3.7. For a semi-simple Frobenius manifold $\left(M, \mathcal{T}_{M}^{f}, g, A\right)$ there holds

(1) Around each point of $M$ there are local coordinates $\left(u_{1}, \ldots, u_{n}\right)$ such that, if $\partial_{i}:=\frac{\partial}{\partial u_{i}}$, then $\partial_{i} \circ \partial_{j}=\delta_{i j} \partial_{i}$.

(2) If the $\left(\nu_{i}\right)$ are the one-forms dual to the $\left(\partial_{i}\right)$, then the metric $g$ and the tensor $A$ have locally the form

$$
\begin{gathered}
g=\sum_{i=1}^{n} h_{i}(u)^{2}\left(\nu_{i}\right)^{2} \\
A=\sum_{i=1}^{n} h_{i}(u)^{2}\left(\nu_{i}\right)^{3} .
\end{gathered}
$$


(3) Let $\gamma_{i j}:=h_{j}^{-1} \partial_{j}\left(h_{i}\right), i \neq j$, be the rotation coefficients of the metric $g$. Then the flatness of $g$ is equivalent to the $\left(\gamma_{i j}\right)$ being a solution of the Darboux-Egoroff system, i.e.

$$
\begin{aligned}
\partial_{k}\left(\gamma_{i j}\right) & =\gamma_{i k} \gamma_{k j}, \quad k \neq i \neq j \neq k, \\
\sum_{k=1}^{n} \partial_{k}\left(\gamma_{i j}\right) & =0, \\
\gamma_{i j} & =\gamma_{j i} .
\end{aligned}
$$

3.8. The linearization of the Darboux-Egoroff system. The system of equations (6) already occurred in the nineteenth century in the work of Darboux on the description of orthogonal curvilinear coordinate systems in $\mathbb{R}^{n}$. It was Egoroff, who gave a complete solution of this problem for symmetric rotation coefficients. According to [Du96], the linearization of the system (6) is

$$
\begin{aligned}
\partial_{k}\left(\psi_{i j}\right) & =\gamma_{i k} \psi_{k j}, \quad k \neq i, \\
\sum_{k=1}^{n} \partial_{k}\left(\psi_{i j}\right) & =0,
\end{aligned}
$$

Moreover he showed in [Du96] that if one has a solution $\left\{\gamma_{i j}\right\}$ of the Darboux-Egoroff system and one $\left\{\psi_{j k}\right\}$ of the associated linear system, then one has locally a solution of the WDVV equations

Theorem 3.9. If the $\left\{\gamma_{i j}\right\}$ satisfy the equations (6) and the $\left\{\psi_{j k}\right\}$ the equations (7), then there corresponds locally a solution of the WDVV-equations to these data. On the domain determined by the inequalities $u_{i} \neq u_{j}, i \neq j$, and $\psi_{11} \ldots \psi_{1 n} \neq 0$, one has the following expressions for the metric $g$ and the tensor $A$

$$
\begin{aligned}
h_{i}(u) & =\psi_{i 1}(u) \\
g_{a b} & =\sum_{k=1}^{n} \psi_{k a}(u) \psi_{k b}(u) \\
A_{a b c} & =\sum_{k=1}^{n} \frac{\psi_{k a}(u) \psi_{k b}(u) \psi_{k c}(u)}{\psi_{k 1}(u)}
\end{aligned}
$$

This is exactly the point where the link between the WDVV-equations and the n-component KPhierarchy will be made. The first step will be to determine the candidate rotation coefficients inside this hierarchy. Next one enforces conditions that will imply the equations of the DE-system and one concludes with finding solutions of the linear system.

\section{Integrable Systems Linked to the WDVV-EQUations}

There are many connections between integrable systems and the WDVV-equations. To give you an impression of what was done in the last decennium, we discuss a number of links and elaborate on one that permits a short description. It all started out with Dubrovin who constructed in [Du92b] for each solution of the WDVV-equation a hierarchy of integrable bihamiltonian equations of hydrodynamic type such that the $\tau$-function of a particular solution of the hierarchy coincides with the genus zero approximation of the corresponding topological field theory model. In [DZ98] the genus one correction to this integrable hierarchy was computed. The remaining connections that we will present go in the opposite direction.

Around the same time Kricever showed in [Kric92a] that certain exact solutions of the KhoklovZabolotskaya hierarchy, which is the hierarchy connected with the dispersionless KP-equation, coincide with the perturbed superpotential of the minimal $A_{n}$ model. This connection was put in a broader perspective in [Kric92b], where it was shown that so-called algebraic orbits in the moduli space of the universal Whitham hierarchy can be identified with various topological matter models coupled with gravity. 
In [LM99] and its sequel [vdL] an orbit of a twisted loop group was shown to yield local Frobenius manifolds. Since this connection can be shown in a comprehensive way without an overload of technical data, we will outline the intermediate steps in the next sections.

The dispersionless limit considered in [Kric92a] was carried out in [Pav] for the generalized DrinfeldSokolov hierarchies, associated to three data: a non twisted affine Lie algebra $\hat{\mathfrak{g}}$, a Heisenberg subalgebra of $\hat{\mathfrak{g}}$ corresponding to a regular primitive conjugacy class in the Weyl group of $\mathfrak{g}$ and a regular element from it. This leads again to algebraic solutions to the WDVV-equations.

A recent construction, see [AvdL], of local Frobenius manifolds starts out with the so-called constrained KP-hierarchy. By using the geometric description of this hierarchy from [HL98] and the additional symmetries from [AGNPZ], they obtain solutions that go beyond the dispersionless limit.

Also the generalized WDVV-equations are intimately linked with integrable systems. For an overview of the various relations and the conjectured ones, we refer to [Mir].

\section{The N-COMponent KP-hierarchy}

5.1. The equations and their linearization. The n-component KP-hierarchy is a matrix version of the KP-hierarchy and the variables involved are $x$ and the $\left\{t_{i \alpha} \| i \geq 1,1 \leq \alpha \leq n\right\}$, all corresponding to a group of commuting flows inherent to the system. A convenient form in which the equations of the n-component KP-hierarchy can be formulated is the so-called Lax form. It consists of a tower of identities between pseudodifferential operators with matrix coefficients, whose coefficients belong to a ring $R$ and we start with a description of this ring. Consider the ring $\mathbb{C}[[x, t]]$ of formal powerseries in $x$ and $t=\left(t_{i \alpha}\right)$. Let $\theta$ be an element in $\mathbb{C}[[x, t]]$ of the form

$$
\theta(x, 0)=x^{N}+\sum_{j>N} a_{j} x^{j}
$$

In the sequel the ring $R$ will always be the localization of $\mathbb{C}[[x, t]]$ w.r.t. the multiplicative subset $S_{\theta}=\left\{\theta^{m} \mid m \geq 0\right\}$ for some $\theta$ in $\mathbb{C}[[x, t]]$ of the form (11). On $R$ we have the derivations $\partial_{i \alpha}=\frac{\partial}{\partial t_{i \alpha}}$ and $\partial=\frac{\partial}{\partial x}$. In the ring $M_{n}(R)[\partial]$ of differential operators in $\partial$ with coefficients from $M_{n}(R)$, one can, in general, not take roots of monic operators. Thereto one passes to the extension $M_{n}(R)\left[\partial, \partial^{-1}\right)$ of all pseudodifferential operators in $\partial$ with coefficients from $M_{n}(R)$. It consists of all expressions

$$
\sum_{i=-\infty}^{N} a_{i} \partial^{i}, a_{i} \in M_{n}(R) \text { for all } i
$$

that are added coefficientwise and that are multiplied according to

$$
\partial^{j} \cdot a \partial^{i}=\sum_{k=0}^{\infty}\left(\begin{array}{c}
j \\
k
\end{array}\right) \partial^{k}(a) \partial^{i+j-k}
$$

Each operator $A=\sum a_{j} \partial^{j}$ decomposes as $A=A_{+}+A_{-}$with $A_{+}=\sum_{j \geq 0} a_{j} \partial^{j}$ its differential operator part and $A_{-}=\sum_{j<0} a_{j} \partial^{j}$ its pure integral operator part. Let $E_{\alpha \beta}$ be the matrix in $M_{n}(R)$ with its $(\alpha \beta)$-entry equal to 1 and the other entries equal to zero. Inside $M_{n}(R)\left[\partial, \partial^{-1}\right)$ we consider operators $L$ and $U_{\alpha}, 1 \leq \alpha \leq n$, of the form

$$
L=\partial+\sum_{j \geq 1} l_{j} \partial^{-j} \text { and } U_{\alpha}=E_{\alpha \alpha}+\sum_{j \geq 1} u_{\alpha, j} \partial^{-j} .
$$

We want them to satisfy the following system of nonlinear equations inside $M_{n}(R)\left[\partial, \partial^{-1}\right)$

$$
\begin{array}{r}
{\left[L, U_{\alpha}\right]=\left[U_{\alpha}, U_{\beta}\right]=0,} \\
\partial_{i \alpha}(L)=\left[\left(L^{i} U_{\alpha}\right)_{+}, L\right], \\
\partial_{i \alpha}\left(U_{\beta}\right)=\left[\left(L^{i} U_{\alpha}\right)_{+}, U_{\beta}\right],
\end{array}
$$


for all $\alpha$ and $\beta$ in $\{1, \ldots, n\}$. These are the equations of the $n$-component $K P$-hierarchy. Given $R$, we call a set of operators $L$ and $U_{\alpha}, 1 \leq \alpha \leq n$, in $M_{n}(R)\left[\partial, \partial^{-1}\right)$ of the form (12) that satisfy these equations, a solution of the $n$-component $K P$-hierarchy. One can see a solution $L$ and $\left\{U_{\alpha}\right\}$ of the $n$-component $K P$-hierarchy as a deformation of the trivial solution $L=\partial$ and $U_{\alpha}=E_{\alpha \alpha}, 1 \leq \alpha \leq n$.

As in the one-dimensional case, there exists a linearization of the $n$-component $K P$-hierarchy from which the Lax equations (14) and (15) follow as compatibility conditions. Consider namely

$$
L \psi=z \psi \quad, \quad U_{\alpha} \psi=\psi E_{\alpha \alpha}
$$

and

$$
\partial_{i \alpha}(\psi)=\left(L^{i} U_{\alpha}\right)_{+}(\psi)=: P_{i \alpha}(\psi) \quad \text { for all } i \geq 1,1 \leq \alpha \leq n .
$$

If one applies the operator $\partial_{i \alpha}$ to both sides of both equations in (16) and performs the following manipulations

$$
\begin{array}{r}
\partial_{i \alpha}(L) \psi+L \partial_{i \alpha}(\psi)=\left\{\partial_{i \alpha}(L)+L P_{i \alpha}\right\} \psi=z \partial_{i \alpha}(\psi)=P_{i \alpha}(z \psi)=\left\{P_{i \alpha} L\right\}(\psi), \\
\partial_{i \alpha}\left(U_{\beta}\right) \psi+U_{\beta} \partial_{i \alpha}(\psi)=\left\{\partial_{i \alpha}\left(U_{\beta}\right)+U_{\beta} P_{i \alpha}\right\} \psi=\partial_{i \alpha}(\psi) E_{\beta \beta}=\left\{P_{i \alpha} U_{\beta}\right\}(\psi),
\end{array}
$$

then one ends up with the equations

$$
\left(\partial_{i \alpha}(L)-\left[\left(L^{i} U_{\alpha}\right)_{+}, L\right]\right)(\psi)=0 \text { and }\left(\partial_{i \alpha}\left(U_{\beta}\right)-\left[\left(L^{i} U_{\alpha}\right)_{+}, U_{\beta}\right]\right)(\psi)=0
$$

So, if we can scratch the function $\psi$ in both expressions, we get the equations (14) and (15) for $L$ and the $\left\{U_{\alpha}\right\}$. Once one has obtained with this procedure operators $L$ and $U_{\alpha}$ that satisfy (14) and (15), one still has to verify the equations (13). This will be immediate from our construction. We proceed now with specifying somewhat more the functions $\psi$ in the linearization. This is done in the next subsection.

5.2. Wavefunctions. From the equations (16) and (17), we see that the functions $\psi$ must be susceptible for left actions of various pseudodifferential operators with coefficients from $M_{n}(R)$ and for right actions with elements from $M_{n}(\mathbb{C})$. Therefore it is not surprising that they will be matrix functions. To motivate the form of the matrix functions $\psi$ that we will choose, we consider the trivial solution $L=\partial$ and $U_{\alpha}=E_{\alpha \alpha}$. Then the equations (16) reduce to

$$
\partial(\psi)=z \psi \quad, \quad E_{\alpha \alpha} \psi=\psi E_{\alpha \alpha} \quad \text { and } \quad \partial_{i \alpha}(\psi)=z^{i} E_{\alpha \alpha} \psi .
$$

Hence, for the trivial solution of the $n$-component $K P$-hierarchy, the matrix function

$$
\gamma(x, t)=\exp \left(x z+\sum_{\alpha=1}^{n} \sum_{i=1}^{\infty} t_{i \alpha} E_{\alpha \alpha} z^{i}\right)=\sum_{k=0}^{\infty} \underline{p}_{k}(x, t) z^{k}
$$

satisfies the equation in (16). Next we introduce a space of matrix functions for which all the manipulations at the end of subsection 5.1 make sense and whose elements can be seen as perturbations of the solution (22). This space $M$ of so-called oscillating matrix functions, is defined as

$$
M=\left\{\left(\sum_{j \leq N} a_{j} z^{j}\right) \exp \left(x z+\sum_{\alpha=1}^{n} \sum_{i=1}^{\infty} t_{i \alpha} E_{\alpha \alpha} z^{i}\right) \mid a_{j} \in M_{n}(R)\right\} .
$$

Note that we will treat in this paper the product in the elements of $M$ of a factor meromorphic at infinity and the exponential factor corresponding to the trivial solution as a formal one. It would no longer be a formal matter, if one can make sense in $M_{n}(R)$ of all the

$$
\sum_{k \in \mathbb{Z}} a_{l-k} \underline{p}_{k}, l \in \mathbb{Z}
$$

and this clearly requires convergence considerations. They have been given in ([HP91]). Here we present a more general algebraic approach. 
The space $M$ can be made into a module for the ring $M_{n}(R)\left[\partial, \partial^{-1}\right)$ of pseudodifferential operators in $\partial$ with coefficients from $M_{n}(R)$. If one puts the actions of the elements $b \in M_{n}(R)$ and the derivations $\partial$ and $\partial_{k \beta}$ as follows

$$
\begin{gathered}
b\left[\left(\sum_{j} a_{j} z^{j}\right) \exp \left(x z+\sum_{\alpha=1}^{n} \sum_{i \geq 1} t_{i \alpha} E_{\alpha \alpha} z^{i}\right)\right]=\left(\sum_{j} b a_{j} z^{j}\right) \gamma(x, t) \\
\partial\left\{\left(\sum_{j} a_{j} z^{j}\right) \exp \left(x z+\sum_{\alpha=1}^{n} \sum_{i \geq 1} t_{i \alpha} E_{\alpha \alpha} z^{i}\right)\right\}=\left(\sum_{j} \partial\left(a_{j}\right)+\sum_{j} a_{j} z^{1+j}\right) \gamma(x, t), \\
\partial_{k \beta}\left\{\left(\sum_{j} a_{j} z^{j}\right) \exp \left(x z+\sum_{\alpha=1}^{n} \sum_{i \geq 1} t_{i \alpha} E_{\alpha \alpha} z^{i}\right)\right\}=\left(\sum_{j} \partial_{k \beta}\left(a_{j}\right)+\sum_{j} a_{j} E_{\beta \beta} z^{k+j}\right) \gamma(x, t),
\end{gathered}
$$

then one sees immediately that $\partial$ acts by an invertible transformation on $M$. Hence these actions extend in a natural way to one of $M_{n}(R)\left[\partial, \partial^{-1}\right)$. Thus $M$ becomes even a free left $M_{n}(R)\left[\partial, \partial^{-1}\right)$-module, for

$$
\left(\sum p_{j} \partial^{j}\right) \cdot \exp \left(x z+\sum_{\alpha, i} t_{i \alpha} E_{\alpha \alpha} z^{i}\right)=\left(\sum p_{j} z^{j}\right) \exp \left(x z+\sum_{\alpha, i} t_{i \alpha} E_{\alpha \alpha} z^{i}\right) .
$$

$M$ is also in a natural way a right module for the diagonal matrices in $M_{n}(\mathbb{C})$. For, if $A=\sum_{i=1}^{n} a_{i} E_{i i}, a_{i} \in$ $\mathbb{C}$, then we put

$$
\left\{\sum a_{j} z^{j}\right\} \exp \left(x z+\sum t_{i \alpha} z^{i} E_{\alpha \alpha}\right) \cdot A:=\left\{\sum_{j} a_{j} A z^{j}\right\} \exp \left(x z+\sum t_{i \alpha} z^{i} E_{\alpha \alpha}\right) .
$$

Since all the operations in the formulae (16) and (17) now make sense in $M$, one can look for solutions of these equations inside $M$. Note that the equations (16) impose still another relation on the operators $U_{\alpha}$. For, we have

$$
\left(\sum_{\alpha=1}^{n} U_{\alpha}\right) \psi=\psi\left(\sum_{\alpha=1}^{n} E_{\alpha \alpha}\right)=\psi
$$

and, since $M$ is a free $M_{n}(R)\left[\partial, \partial^{-1}\right)$-module, this implies that

$$
\sum_{\alpha=1}^{n} U_{\alpha}=I d
$$

Hence the solutions of the $n$-component $K P$-hierarchy that one obtains via the linearization satisfy still this additional condition.

There are two types of transformations that map solutions $\psi$ in $M$ of the equations (16) and (17) into new ones for the same operators $L$ and $U_{\alpha}$. First there is the right action of the diagonal matrices in $M_{n}(\mathbb{C})$. Secondly there is an action of a discrete group. For, if $\delta=\left(\delta_{1}, \ldots, \delta_{n}\right) \in \mathbb{Z}^{n}$ and we write $z^{\delta}$ for the element

$$
\operatorname{diag}\left(z^{\delta_{1}}, \ldots, z^{\delta_{n}}\right)=\left(\begin{array}{ccc}
z^{\delta_{1}} & & 0 \\
& \ddots & \\
0 & & z^{\delta_{n}}
\end{array}\right) .
$$

Then the element $z^{\delta} \exp \left(\sum t_{i \alpha} z^{i} E_{\alpha \alpha}\right)$ belongs to $M$ and we have

$$
z^{\delta} \gamma(x, t)=\left(\sum_{\alpha=1}^{n} E_{\alpha \alpha} \partial_{1 \alpha}^{\delta_{\alpha}}\right) \exp \left(x z+\sum t_{i \alpha} z^{i} E_{\alpha \alpha}\right)=: R(\delta) \cdot \gamma(x, t)
$$


Clearly the element $R(\delta)$ in $M_{n}(R)\left[\partial, \partial^{-1}\right)$ is invertible with inverse $R(\delta)^{-1}=R(-\delta)$. Note that, if $\psi=P \cdot \exp \left(x z+\sum t_{i \alpha} z^{i} E_{\alpha \alpha}\right) \in M$ satisfies the equations in (16) and (17), then also

$$
\psi^{(\delta)}=P \cdot R(\delta) \cdot \exp \left(x z+\sum t_{i \alpha} z^{i} E_{\alpha \alpha}\right) .
$$

Any matrix function $\psi$ in $M$ can without any restriction be written in the form (26) with $P$ of the form $P=\sum_{j \leq 0} p_{j} \partial^{j}$. We assume now that the leading coefficient of $P$ is equal to the identity. This gauge carries a special name: an element $\psi$ in $M$ is called an oscillating matrix function of type $z^{\delta}$ if it has the form

$$
\psi=P \cdot z^{\delta} \gamma(t)=P \cdot R(\delta) \cdot \exp \left(x z+\sum t_{i \alpha} z^{i} E_{\alpha \alpha}\right) \text { with } P=\operatorname{Id}+\sum_{j<0} p_{j} \partial^{j} .
$$

The connection between oscillating matrix functions of type $z^{\delta}$ satisfying (16) and (17) and solutions of the $n$-component $K P$-hierarchy is given by

Proposition 5.3. Let $\psi=P \cdot R(\delta) \cdot \exp \left(x z+\sum t_{i \alpha} z^{i} E_{\alpha \alpha}\right)$ in $M$ be an oscillating matrix function of type $z^{\delta}$. Assume, we have operators $L$ and $U_{\alpha}, 1 \leq \alpha \leq n$, in $M_{n}(R)\left[\partial, \partial^{-1}\right)$ of the form (12), such that the equations in (16) hold and such that for $i \geq 1$ and all $\beta, 1 \leq \beta \leq n$, there holds

$$
\partial_{i \beta}(\psi)=B_{i \beta}(\psi) \text { for some } B_{i \beta} \in M_{n}(R)[\partial] \text {. }
$$

Then we have $L=P \partial P^{-1}, U_{\alpha}=P E_{\alpha \alpha} P^{-1}$ and $B_{i \beta}=P_{i \beta}=\left(L^{i} U_{\beta}\right)_{+}$for all $i$ and $\beta$. Moreover the operators $L$ and the $\left\{U_{\alpha}\right\}$ are a solution of the n-component KP-hierarchy.

For a proof we refer the reader to [HL00]. A useful consequence of this proposition is

Corollary 5.4. An oscillating matrix function $\psi=P R(\delta) \exp \left(x z+\sum t_{i \alpha} z^{i} E_{\alpha \alpha}\right)$ of type $z^{\delta}$, satisfies equation (17) if and only if $P$ satisfies the so-called Sato-Wilson equations:

$$
\partial_{i \alpha}(P) P^{-1}=-\left(P \partial^{i} E_{\alpha \alpha} P^{-1}\right)_{-}, \text {for all } i \geq 1,1 \leq \alpha \leq n .
$$

Such a function $\psi$ is called a wavefunction of the n-component KP-hierarchy.

5.5. The bilinear equations. At the description of the bilinear form of the $n$-component $K P$-hierarchy and at the construction of Darboux transformations of this system of equations it is convenient to have besides $M$ also at one's disposal its adjoint space $M^{*}$ consisting of all formal products

$$
\left\{\sum_{j \leq N} a_{j} z^{j}\right\} \exp \left(-x z-\sum_{\alpha=1}^{n} \sum_{i=1}^{\infty} t_{i \alpha} z^{i} E_{\alpha \alpha}\right)=\left\{\sum_{j \leq N} a_{j} z^{j}\right\} \gamma(-x,-t),
$$

where $a_{j} \in M_{n}(R)$ for all $j$. On the space $M^{*}$ we define the following actions of $M_{n}(R), \partial$ and the $\left\{\partial_{k \beta}\right\}$

$$
\begin{aligned}
b\left\{\left(\sum_{j} a_{j} z^{j}\right) \gamma(-x,-t)\right\} & =\left(\sum_{j} b a_{j} z^{j}\right) \gamma(-x,-t) \\
\partial_{k \beta}\left\{\left(\sum_{j} a_{j} z^{j}\right) \gamma(-x,-t)\right\} & =\left(\sum_{j} \partial_{k \beta}\left(a_{j}\right) z^{j}-\sum_{j} a_{j} E_{\beta \beta} z^{k+j}\right) \gamma(-x,-t) \\
\partial\left\{\left(\sum_{j} a_{j} z^{j}\right) \gamma(-x,-t)\right\} & =\left(\sum_{j} \partial_{k \beta}\left(a_{j}\right) z^{j}-\sum_{j} a_{j} z^{1+j}\right) \gamma(-x,-t)
\end{aligned}
$$

In particular we see that $\partial$ acts invertible on $M^{*}$, so that the natural extension of these actions leads to an $M_{n}(R)\left[\partial, \partial^{-1}\right)$-module structure on $M^{*}$, which is again free, since we have

$$
\sum_{j} P_{j}(-\partial)^{j} \cdot \exp \left(-x z-\sum t_{i \alpha} z^{i} E_{\alpha \alpha}\right)=\left\{\sum_{j} P_{j} z^{j}\right\} \exp \left(-x z-\sum t_{i \alpha} z^{i} E_{\alpha \alpha}\right) .
$$

Now we have a bilinear pairing $\mathcal{R}: M \times M^{*} \rightarrow M_{n}(R)$ defined as follows: if $\varphi=\varphi(x, t, z)=$ $\left(\sum_{j} a_{j}(x, t) z^{j}\right) \exp \left(x z+\sum t_{i \alpha} z^{i} E_{\alpha \alpha}\right)$ belongs to $M$ and $\psi=\psi(x, t, z)=\left(\sum_{k} b_{k}(x, t) z^{k}\right) \exp (-x z-$ 
$\left.\sum t_{i \alpha} z^{i} E_{\alpha \alpha}\right)$ is an element of $M^{*}$, then we put

$$
\begin{aligned}
\mathcal{R}(\varphi, \psi): & =\operatorname{Res}_{z}\left(\left(\sum_{j} a_{j}(x, t) z^{j}\right)\left(\sum b_{k}(x, t)^{T} z^{k}\right)\right) \\
& =\sum_{k \in \mathbb{Z}} a_{-k-1}(x, t) b_{k}(x, t)^{T}
\end{aligned}
$$

where $a^{T}$ for each a in $M_{n}(R)$ denotes the transposed of $a$. Clearly the defining sum in $\mathcal{R}$ is finite, hence belongs to $M_{n}(R)$. On the algebra $M_{n}(R)\left[\partial, \partial^{-1}\right)$ we have a $\mathbb{C}$-linear anti-algebra morphism called "taking the adjoint". The adjoint of $P=\sum P_{j} \partial^{j}$ is given by

$$
\begin{aligned}
P^{*} & =\sum_{j}(-\partial)^{j} P_{j}^{T}=\sum_{j}(-1)^{j} \sum_{k \geq 0}\left(\begin{array}{c}
j \\
k
\end{array}\right) \partial^{k}\left(P_{j}^{T}\right) \partial^{j-k} \\
& =\sum_{l}\left\{\sum_{k \geq 0}(-1)^{l+k}\left(\begin{array}{c}
l+k \\
k
\end{array}\right) \partial^{k}\left(P_{l+k}^{T}\right)\right\} \partial^{l} .
\end{aligned}
$$

The following theorem gives an important connection between the bilinear form $\mathcal{R}$ and taking the adjoint. Its proof can be found in [HLOO]

Theorem 5.6. Let $\varphi(x, t, z)=P(x, t, \partial) \cdot \exp \left(x z+\sum t_{i \alpha} z^{i} E_{\alpha \alpha}\right) \in M$ and $\psi(t, z)=Q(x, t, \partial) \cdot \exp (-x z-$ $\left.\sum t_{i \alpha} z^{i} E_{\alpha \alpha}\right) \in M^{*}$, then

$$
\begin{aligned}
\left(P(x, t, \partial) Q(x, t, \partial)^{*}\right)_{-} & =-\sum_{n=0}^{\infty} \mathcal{R}\left(\varphi(x, t, z), \partial^{n}(\psi(x, t, z))\right)(-\partial)^{-n-1} \\
& =: \mathcal{R}\left(\varphi(x, t, z), \partial^{-1} \circ \psi(x, t, z)\right)
\end{aligned}
$$

Like we have in $M$ the notion of oscillating matrix function of type $z^{\delta}$, we have in $M^{*}$ that of dual oscillating matrix function of type $z^{-\delta}$. This is by definition an element $\psi$ in $M^{*}$ of the form

$$
\begin{aligned}
\psi(x, t, z) & =Q(x, t, z) \cdot R(-\delta)^{*} \cdot \exp \left(-x z-\sum t_{i \alpha} z^{i} E_{\alpha \alpha}\right) \\
& =\left\{\left(1+\sum_{j<0} g_{j} z^{j}\right) z^{-\delta}\right\} \exp \left(-x z-\sum t_{i \alpha} z^{i} E_{\alpha \alpha}\right)
\end{aligned}
$$

If $\varphi=P(x, t, \partial) R(\delta) \cdot \exp \left(x z+\sum t_{i \alpha} z^{i} E_{\alpha \alpha}\right)$ in $M$ is an oscillating matrix function of type $z^{\delta}$, then

$$
\varphi^{*}=\left(P(t, \partial)^{*}\right)^{-1} R(-\delta)^{*} \cdot \exp \left(-x z-\sum t_{i \alpha} z^{i} E_{\alpha \alpha}\right)
$$

is clearly a dual oscillating matrix function of type $z^{-\delta}$. It is called the adjoint of $\varphi$. Since

$$
\left(P(x, t, \partial)\left(\left(P(x, t, \partial)^{*}\right)^{-1}\right)^{*}\right)_{-}=0,
$$

the foregoing theorem shows that for all $n \geq 0$

$$
\mathcal{R}\left(\varphi, \partial^{n}\left(\varphi^{*}\right)\right)=0 \text {. }
$$

This property even characterizes $\varphi^{*}$ among the dual oscillating matrix functions of type $z^{-\delta}$. For, if $\psi=Q(x, t, \partial) R(-\delta)^{*} \exp \left(-x z-\sum t_{i \alpha} z^{i} E_{\alpha \alpha}\right) \in M^{*}$ is such a function satisfying (30) with $\varphi^{*}$ replaced by $\psi$, then we have according to the theorem

$$
P R(\delta)\left(R(-\delta)^{*}\right)^{*} Q^{*}=P Q^{*}=\partial^{0}+\left(P Q^{*}\right)_{-}=1
$$

In other words, $Q=\left(P^{*}\right)^{-1}$ and $\psi$ is the adjoint of $\varphi$. We will use this criterion later on and therefore we resume it in a

Corollary 5.7. Let $\varphi$ be an oscillating matrix function of type $z^{\delta}$ and let $\psi$ be a dual oscillating matrix function of type $z^{-\delta}$. Then $\psi$ is the adjoint of $\varphi$ if and only if it satisfies

$$
\mathcal{R}\left(\varphi(x, t, z), \partial^{n}(\psi(x, t, z))\right)=0 \text { for all } n \geq 0 \text {. }
$$


If $\varphi$ is a wavefunction of the $n$-component $K P$-hierarchy, then its adjoint $\varphi^{*}$ is called a dual wavefunction of the $n$-component $K P$-hierarchy. It satisfies a set of linear equations similar to (17), see [HLO0].

The bilinear form $\mathcal{R}$ plays also an important role at the interpretation of the famous bilinear identities for an oscillating function $\psi$ and and its dual, (see [DJKM]):

$$
\operatorname{Res}_{z} \psi(x, t, z) \psi^{*}(y, s, z)^{T}=0
$$

First we have a look at the formal product $\psi(x, t, z) \phi(x, s, z)^{T}$, where $\psi(x, t, z)=\left\{\sum_{k} a_{k}(x, t) z^{k}\right\} \gamma(x, t) \in$ $M$ and $\phi(y, t, z)=\left\{\sum_{m} b_{m}(y, s) z^{m}\right\} \gamma(-y,-t) \in M^{*}$. If we write $e^{x z+\sum t_{i \alpha} z^{i} E_{\alpha \alpha}}=\sum_{r=0}^{\infty} p_{r}\left(x,\left(t_{i \alpha}\right)\right) z^{r}$, then this formal product becomes

$$
\left(\sum_{k} a_{k}(x, t) z^{k}\right)\left(\sum_{r=0}^{\infty} p_{r}\left(x-y,\left(t_{i \alpha}-s_{i \alpha}\right)\right) z^{r}\right)\left(\sum_{m} b_{m}(y, s)^{T} z^{m}\right)
$$

Hence the equation (31) for $\phi=\psi^{*}$ boils formally down to

$$
\sum_{r=0}^{\infty} \sum_{k} a_{k}(x, t) p_{r}\left(x-y,\left(t_{i \alpha}-s_{i \alpha}\right)\right) b_{-r-1-k}(y, s)^{T}=0,
$$

for all relevant $t=\left(t_{i \alpha}\right)$ and $s=\left(s_{i \alpha}\right)$. In order to avoid convergence considerations, we look at a few differential consequences of these relations that always exist. If one differentiates the equation namely with respect to $x$ or some of the parameters $\left(t_{i \alpha}\right)$ or $\left(s_{i \alpha}\right)$ and substitutes next $t_{i \alpha}=s_{i \alpha}$ for all $i \geq 1$, then one ends up with finite expressions in the coefficients of $\psi$ and $\psi^{*}$. Thus we get for example for all $k \geq 0, i \geq 1,1 \leq \alpha \leq n$, and all $m \geq 0$ the relations

$$
\begin{aligned}
\mathcal{R}\left(\partial^{k}(\psi(x, t, z)), \partial^{m}\left(\psi^{*}(x, t, z)\right)\right) & =0, \\
\mathcal{R}\left(\partial_{i \alpha}(\psi(x, t, z)), \partial^{m}\left(\psi^{*}(x, t, z)\right)\right) & =0 .
\end{aligned}
$$

Note that this first equation can also be obtained by applying $\partial$ several times to the relations (30). Assume now that an oscillating matrix function $\psi$ of type $z^{\delta}$ and its dual $\psi^{*}$ satisfy the equations (33) and (34). Let $L=P \partial P^{-1}$ and $U_{\alpha}=P E_{\alpha \alpha} P^{-1}$ be the operators associated with $\psi$. From the form of the action of the $\partial_{i \alpha}$ on $\psi$ one sees that there exists for each $i \geq 1$ and all $\alpha, 1 \leq \alpha \leq n$, an differential operator $Q_{i \alpha}$ in $M_{n}(R)[\partial]$ of degree $i$ such that

$$
\partial_{i \alpha}(\psi)-Q_{i \alpha}(\psi)=G_{i \alpha} \partial^{l} e^{x z+\sum_{i} t_{i} z^{i}} \text {, with } G_{i \alpha} \in M_{n}(R)\left[\partial, \partial^{-1}\right) \text { of degree }<0 .
$$

Because of the relations in (33) and (34) we have then for all $m \geq 0$ that

$$
\mathcal{R}\left(\partial_{i \alpha}(\psi(x, t, z))-\left(Q_{i \alpha}\right)(\psi(x, t, z)), \partial^{m}\left(\psi^{*}(x, t, z)\right)\right)=0 .
$$

According to theorem 5.6, we get then that $\left(G_{i \alpha} \partial^{l} P^{-1}\right)_{-}=G_{i \alpha} \partial^{l} P^{-1}=0$. Thus we have obtained that $\partial_{i \alpha}(\psi(x, t, z))-\left(Q_{i \alpha}\right)(\psi(x, t, z))=0$. From proposition 5.3 we know then that this implies for all $i \geq 1$ and all $\alpha$ that the operator $Q_{i \alpha}$ is equal to $\left(L^{i} U_{\alpha}\right)_{+}$. Hence $\psi$ is a wavefunction of the n-component $K P$-hierarchy. Reversely, if $\psi$ is a wavefunction of the the n-component $K P$-hierarchy, then the first equation in (33) holds and since $\partial_{i \alpha}$ acts on $\psi$ as $\left(L^{i} U_{\alpha}\right)+$ also the second relation holds. Thus we have found the following characterization of the wavefunctions in $M$

Proposition 5.8. An oscillating matrix function $\psi$ of type $z^{l}$ is a wavefunction of the n-component $K P$-hierarchy if and only if $\psi$ and its dual $\psi^{*}$ satisfy the equations (33) and (34).

A consequence of this proposition is that wavefunctions of the n-component $K P$-hierarchy are characterized by the following set of equations

$$
\mathfrak{R}\left(\Delta_{1}(\psi(x, t, z)), \Delta_{2}\left(\psi^{*}(x, t, z)\right)\right)=0,
$$

where $\Delta_{1}$ and $\Delta_{2}$ are arbitrary finite products of the $\left\{\partial_{i \alpha}\right\}$ and $\partial$. These equations are the algebraic version of the equations (31). 
5.9. Grassmannian construction of solutions. In this subsection we describe the Sato Grassmannian, from which one can construct matrix wavefunctions of the $n$-component KP-hierarchy. It is a vector form of the line set out in [?]. Since the rows of these functions consist of infinite series in $z$ and $z^{-1}$ with coefficients from $\mathbb{C}^{n}$, it is not so strange that the basic manifold for the relevant subspaces consists of a class of series in $z$ and $z^{-1}$. Consider the spaces

$$
\begin{aligned}
& H_{-}=\left(z^{-1} \mathbb{C}\left[\left[z^{-1}\right]\right]\right)^{n}=\left\{\sum_{j=1}^{\infty} a_{j} z^{-j} \mid a_{j} \in \mathbb{C}^{n}\right\} \quad \text { and } \\
& H_{+}=(\mathbb{C}[z])^{n}=\left\{\sum_{i=0}^{m} b_{i} z^{i} \mid b_{i} \in \mathbb{C}^{n}\right\} .
\end{aligned}
$$

Hence $H=H_{+} \oplus H_{-}$is equal to $\left(\mathbb{C}\left(\left(z^{-1}\right)\right)\right)^{n}$, where $\mathbb{C}\left(\left(z^{-1}\right)\right)$ is the quotient field of $\mathbb{C}\left[\left[z^{-1}\right]\right]$. First we fix some notations in the space $H$. For $l, 0 \leq l \leq n-1$, let $f_{l}$ be the vector in $\mathbb{C}^{n}$ with a one at the $l+1$-th place and elsewhere zeros. Then we denote for all $m \in \mathbb{Z}$ and all $l, 0 \leq l \leq n-1$, the vector $f_{l} z^{m}$ by $e_{l+m n}$. Inside $H$ we consider the subspaces $H_{k}, k \in \mathbb{Z}$ given by

$$
H_{k}:=\left\{\sum_{r \geq k} a_{r} e_{r}, \text { with } a_{r} \in \mathbb{C} \text { and } \sum_{r \geq k} a_{r} e_{r} \in H\right\} .
$$

For each $k \in \mathbb{Z}$ let $p_{k}: H \rightarrow H_{k}$ be the projection

$$
p_{k}\left(\sum a_{j} e_{j}\right)=\sum_{j \geq k} a_{j} e_{k} .
$$

Then the Grassmann manifold of Sato consists of all subspaces of $H$ that are of a size comparable to $H_{+}$. More precisely, it is given by

$$
\operatorname{Gr}(H)=\left\{\begin{array}{l|l}
W & \begin{array}{l}
W \subset H, p_{0}: W \rightarrow H_{+} \text {has a finite } \\
\text { dimensional kernel and cokernel }
\end{array}
\end{array}\right\}
$$

The space $\operatorname{Gr}(H)$ has a subdivision into different components according to the index of $\left.p_{0}\right|_{W}$ that is defined by

$$
\text { ind }\left(\left.p_{0}\right|_{W}\right)=\operatorname{dim}\left(\operatorname{Ker}\left(\left.p_{0}\right|_{W}\right)\right)-\operatorname{dim}\left(\operatorname{Coker}\left(\left.p_{0}\right|_{W}\right)\right) .
$$

We denote these components as follows

$$
\operatorname{Gr}^{(k)}(H)=\left\{W \mid W \in \operatorname{Gr}(H), \operatorname{ind}\left(\left.p_{0}\right|_{W}\right)=-k\right\} .
$$

Clearly, the subspace $H_{k}$ belongs to $\operatorname{Gr}^{(k)}(H)$ and one easily verifies that the component $\operatorname{Gr}^{(k)}(H)$ can also be described as

$$
\operatorname{Gr}^{(k)}(H)=\left\{W \mid W \in \operatorname{Gr}(H), \operatorname{ind}\left(\left.p_{k}\right|_{W}\right)=0\right\} .
$$

The "big cell" in $\operatorname{Gr}^{(k)}(H)$ consists of all subspaces in $\operatorname{Gr}(H)$ that project bijectively onto $H_{k}$, i.e. all $W$ in

$$
O^{(k)}=\left\{\begin{array}{l|l}
W & \begin{array}{l}
W \in \mathrm{Gr}(H), w \mapsto p_{k}(w) \\
\text { bijection: } W \rightarrow H_{k}
\end{array}
\end{array}\right\}
$$

On the space $H$ we have a bilinear form $B$ that plays a role in the sequel. Namely, if $f=\sum_{j} a_{j} z^{j}$ and $g=\sum_{j} b_{j} z^{j}$ are in $H$, then we define

$$
B(f, g)=\operatorname{Res}_{z}(f(z) g(z))=\sum_{j} a_{j} b_{-j-1}^{T},
$$

where we have written the elements of $\mathbb{C}^{n}$ as rows and $A^{T}$ denotes the transposed of a matrix $A$. For $W$ in $\operatorname{Gr}(H)$, let $W^{\perp}$ be the orthocomplement of $W$ in $H$ w.r.t.this form $B$. With the above given description of spaces in $\operatorname{Gr}(H)$ one verifies that $W^{\perp}$ also belongs to $\operatorname{Gr}(H)$. 
Our next step will be to show how the subspaces from $\operatorname{Gr}(H)$ occur as the span of the rows of the Laurent coefficients of certain oscillating functions. Consider a matrix wavefunction $\psi$ of the $n$ component $K P$-hierarchy and its dual $\psi^{*}$ that have the form

$$
\begin{aligned}
\psi(x, t, z) & =\left\{\sum_{j \leq 0} a_{j}(x, t) z^{j}\right\} z^{\delta} \exp \left(x z+\sum t_{i \alpha} z^{i} E_{\alpha \alpha}\right) \\
\psi^{*}(x, s, z) & =\left\{\sum_{m \leq 0} b_{m}(x, t) z^{m}\right\} z^{-\delta} \exp \left(-x z-\sum t_{i \alpha} z^{i} E_{\alpha \alpha}\right) .
\end{aligned}
$$

The class of wavefunctions we will consider in this paper that satisfies the condition that there is an $\theta$ in $\mathbb{C}[[x, t]]$ of the form (11) such that for all $m \leq 0$ and all $j \leq 0$

$$
\theta(x, t) a_{j}(x, t) \in M_{n}(\mathbb{C}[[x, t]]) \text { and } \theta(x, t) b_{m}(x, t) \in M_{n}(\mathbb{C}[[x, t]])
$$

These matrix wavefunctions are called regularizable. For regularizable wavefunctions the Laurent series in $x$ of $\psi$ and $\psi^{*}$ have the form

$$
\psi(x, t, z)=\sum_{j \geq-N} w_{j}(t, z) x^{j} \exp \left(x z+\sum t_{i \alpha} z^{i} E_{\alpha \alpha}\right),
$$

where $w_{j}(t, z)=\sum_{l=-\infty}^{N_{1}} v_{l} z^{l}$, with $v_{l} \in \mathbb{C}[[t]]^{n}$,

$$
\psi^{*}(x, t, z)=\sum_{j \geq-N} w_{j}^{*}(t, z) x^{j} \exp \left(-x z-\sum t_{i \alpha} z^{i} E_{\alpha \alpha}\right),
$$

where $w_{j}^{*}(t, z)=\sum_{l=-\infty}^{N_{2}} v_{l}^{*} z^{l}$, with $v_{l}^{*} \in \mathbb{C}[[t]]^{n}$. It is not hard to show that both spaces

$$
W=\operatorname{Span}\left\{\operatorname{rows} \text { of } w_{j}(0, z), j \geq-N\right\} \text { and } W^{*}=\operatorname{Span}\left\{\operatorname{rows} \text { of } w_{j}^{*}(0, z), j \geq-N\right\}
$$

belong to $\operatorname{Gr}(H)$. Following ideas of Sato, one can show, see [DJKM], that the space $W$ even determines $\psi$, for there holds

Proposition 5.10. The map $B_{\delta}$ that associates to a regularizable wavefunction $\psi$ of type $z^{\delta}$ of the $n$-component KP-hierarchy the span of the rows of the coefficients in $t=0$ of the Laurent series of $\psi$ in $x$ is an injection from the class of matrix wavefunctions of type $z^{\delta}$ into $\operatorname{Gr}(H)$. All planes in $\operatorname{Gr}(H)$ occur in the image of this map for a certain type $z^{\delta}$. The same properties hold for the map $B_{\delta}^{*}$ that associates to a regularizable dual wavefunction $\psi^{*}$ of type $z^{-\delta}$ of the $n$-component KP-hierarchy the span of the rows of the coefficients in $t=0$ of the Laurent series of $\psi^{*}$ in $x$.

It might happen that there correspond to a certain plane, matrix wavefunctions of various types, see [HP94]. The wavefunctions that satisfy the conditions in (41) for $\theta=1$ correspond to the union of the big cells. For each $z^{\delta}$ and each $W \in \operatorname{Gr}(H)$ in the image of $B_{\delta}$, we write the matrix wavefunction of type $z^{\delta}$ corresponding to $W$ and its dual matrix wavefunction as

$$
\begin{aligned}
\psi_{W}^{(\delta)} & =\hat{\psi}_{W}^{(\delta)} z^{\delta} \exp \left(x z+\sum t_{i \alpha} z^{i} E_{\alpha \alpha}\right) \text { resp. } \\
\left(\psi_{W}^{(\delta)}\right)^{*} & =\left(\hat{\psi}_{W}^{(\delta)}\right)^{*} z^{-\delta} \exp \left(-x z-\sum t_{i \alpha} z^{i} E_{\alpha \alpha}\right) .
\end{aligned}
$$

Now that we have this link between matrix wavefunctions and the Grassmann manifold, we can also give a geometric description of the dual wavefunction of $\psi_{W}^{(\delta)}$. From the characterizing properties in (33), follows

Proposition 5.11. Let $W$ belong to the image of $B_{\delta}$ and let $\tilde{W}$ be a subspace in $\operatorname{Gr}(H)$. Then $\tilde{W}$ is the space $W^{*}$ corresponding to the dual wavefunction $\left(\psi_{W}^{(\delta)}\right)^{*}$, if and only if $\tilde{W}=W^{\perp}$ with $W^{\perp}$ the orthocomplement of $W$ w.r.t. the bilinear form $B$ on $H$. 


\section{From N-COMPONENT KP TO WDVV}

6.1. The relation $\partial_{\mathbf{k}}\left(\gamma_{\mathbf{i j}}\right)=\gamma_{\mathbf{i k}} \gamma_{\mathbf{k} \mathbf{j}}$. Let $\psi=P \cdot z^{\delta} \gamma(t)=P \cdot R(\delta) \cdot \exp \left(x z+\sum t_{i \alpha} z^{i} E_{\alpha \alpha}\right)$ with $P=$ $\mathrm{Id}+\sum_{j<0} p_{j} \partial^{j}$ be a wavefunction of the n-component KP-hierarchy. Then we know from 5.4 that $P$ satisfies the Sato equations

$$
\begin{aligned}
\partial_{i \alpha}(P) & =-\left(P \partial^{i} E_{\alpha \alpha} P^{-1}\right)_{-} P \\
& =-P \partial^{i} E_{\alpha \alpha}+\left(P \partial^{i} E_{\alpha \alpha} P^{-1}\right)_{+} P .
\end{aligned}
$$

We want to show now that the coefficients of $p_{-1}$ are the candidate rotation coefficients and that the $\left\{t_{1 \alpha}\right\}$ will serve as the coordinates $u_{\alpha}$ from theorem 3.7. A direct computation shows that

$$
\left(P \partial E_{\alpha \alpha} P^{-1}\right)_{+}=\partial E_{\alpha \alpha}+\left[p_{-1}, E_{\alpha \alpha}\right] .
$$

Substituting this in the Sato equation for $i=1$ and looking at the coefficient of $\partial^{-1}$ results in the matrix equality

$$
\partial_{i \alpha}\left(p_{-1}\right)=\left[E_{\alpha \alpha}, p_{-2}\right]+E_{\alpha \alpha} \partial\left(p_{-1}\right)+\left[p_{-1}, E_{\alpha \alpha}\right] p_{-1} .
$$

This relation implies for the off-diagonal entries of $p_{-1}$ that for $\alpha \neq \beta \neq \gamma \neq \alpha$

$$
\partial_{i \alpha}\left(\left(p_{-1}\right)_{\beta \gamma}\right)=0+0+\left(p_{-1}\right)_{\beta \alpha}\left(p_{-1}\right)_{\alpha \gamma}+0,
$$

which is exactly the relation we are looking for.

6.2. The dilatation invariance. Now that we have our candidate rotation coefficients, the $\left(p_{-1}\right)_{\beta \alpha}, \beta \neq$ $\alpha$, and know that $u_{\alpha}=t_{1 \alpha}$, we have $\sum_{k} \partial_{k}=\sum_{\alpha} \partial_{i \alpha}=\partial$. The action of $\partial$ on $\psi$ is

$$
\partial(\psi)=\left\{\sum_{i<0} \partial\left(p_{i}\right) z^{i}\right\} z^{\delta} \gamma(x, t, z)+\left\{\sum_{i<0} p_{i} z^{i+1}\right\} z^{\delta} \gamma(x, t, z) .
$$

Hence we see that the condition $\partial(\psi)=z \psi$ is equivalent to $\partial\left(p_{i}\right)=0$ for all $i<0$. This is more than sufficient to get the dilatation invariance. Let $W=B_{\delta}(\psi)$. Then it is well-known that the relation $\partial(\psi)=z \psi$, amounts to the geometric property $z W \subset W$. Examples of such subspaces are the

$$
W=g(z) H_{+}, \text {where } g(z) \in L\left(S^{1}, G l_{n}(\mathbb{C})\right),
$$

the loopgroup of $G l_{n}(\mathbb{C})$.

6.3. The symmetry of the rotation coefficients. To get the symmetry of the rotation coefficients we have to restrain the planes from the Grassmannian still more. Thereto we consider here besides the wavefunction $\psi$ also its dual wavefunction

$$
\psi^{*}=\left(P^{*}\right)^{-1} . z^{-\delta} \gamma(-x,-t, z)=\left\{I d-p_{-1}^{T} z^{-1}+\ldots\right\} z^{-\delta} \gamma(-x,-t, z)
$$

Compare this function with

$$
\psi(-x, t,-z)=\left\{\sum_{i}(-1)^{i} p_{i} z^{i}\right\}(-z)^{\delta} \exp \left(-x z+\sum_{\alpha=1}^{n} \sum_{i=1}^{\infty}(-1)^{i} t_{i \alpha} E_{\alpha \alpha} z^{i}\right) .
$$

Then we see that, if we choose $\delta=0, \tilde{t}=\left(t_{1 \alpha}, 0, t_{3 \alpha}, 0, \ldots\right)$, the equality

$$
\psi(-x, \tilde{t},-z)=\psi^{*}(x, \tilde{t}, z)
$$

implies that $p_{-1}=p_{-1}^{T}$ and this is the result we want. Recall that the dual wavefunction was characterized by the bilinear equations from 5.7. Thus one obtains the equivalence

$$
\mathcal{R}\left(\psi(x, \tilde{t}, z), \partial^{n}(\psi(-x, \tilde{t},-z))\right)=0 \text { for all } n \geq 0 \Leftrightarrow \psi(-x, \tilde{t},-z)=\psi^{*}(x, \tilde{t}, z) .
$$

By using this characterization, one verifies that all the

$$
W=g(z) H_{+}, g(z) \in L\left(S^{1}, G l_{n}(\mathbb{C})\right) \text { and } g(z)^{-1}=g(-z)^{T},
$$

satisfy these conditions and hence supply a large collection of solutions of the Darboux-Egoroff equations. 
6.4. The linearization. In view of the foregoing results we start with a plane $W=g(z) H_{+}, g(z) \in$ $L\left(S^{1}, G l_{n}(\mathbb{C})\right)$ and $g(z)^{-1}=g(-z)^{T}$. The corresponding wavefunction of type $I d$ satisfies the equations

$$
\begin{aligned}
\sum_{\alpha=1}^{n} \partial_{1 \alpha} \psi & =z \psi \\
\partial_{1 \alpha}(\psi) & =\left\{z E_{\alpha \alpha}+\left[p_{-1}, E_{\alpha \alpha}\right]\right\} \psi .
\end{aligned}
$$

Like in the foregoing section we put the even numbered flows equal to zero and introduce the function

$$
\theta(x, \tilde{t}, z):=z^{-1} \psi(x, \tilde{t}, z) g(z) .
$$

It satisfies the same equations as $\psi$

$$
\begin{aligned}
\sum_{\alpha=1}^{n} \partial_{1 \alpha}(\theta(x, \tilde{t}, z)) & =z \theta(x, \tilde{t}, z) \\
\partial_{1 \alpha}(\theta(x, \tilde{t}, z)) & =\left\{z E_{\alpha \alpha}+\left[p_{-1}, E_{\alpha \alpha}\right]\right\} \theta(x, \tilde{t}, z) .
\end{aligned}
$$

From the bilinear relations one deduces, see [vdL],

Proposition 6.5. For the function $\theta(x, \tilde{t}, z)$ hold the following matrix identities

$$
\operatorname{Res}_{z}(\theta(x, \tilde{t}, z)) \neq 0 \text { and } \operatorname{Res}_{z}(z \theta(x, \tilde{t}, z))=0 .
$$

The first says that, if we define $\Psi(x, \tilde{t}):=\operatorname{Res}_{z}(\theta(x, \tilde{t}, z))=\left(\Psi_{i j}(x, \tilde{t})\right)$, this is a nonzero matrix function. Substitution of the second property in the equations (51) gives that the $\left(\Psi_{i j}(\tilde{t})\right)$ satisfy

$$
\begin{aligned}
\sum_{\alpha=1}^{n} \partial_{1 \alpha}\left(\Psi_{i j}(x, \tilde{t})\right) & =0 \\
\partial_{1 \alpha}\left(\Psi_{i j}\right) & =\sum_{s}\left[p_{-1}, E_{\alpha \alpha}\right]_{i s} \Psi_{s j}=\left(p_{-1}\right)_{i \alpha} \Psi_{\alpha j}, \alpha \neq i .
\end{aligned}
$$

In other words they are a solution of the linearization of the Darboux-Egoroff equations for the rotation coefficients $\left(p_{-1}\right)_{i j}$.

\section{REFERENCES}

[AGNPZ] H. Aratyn, J. F. Gomes, E. Nissimov, S. Pacheva, A. H. Zimmerman, Symmetry flows, conservation laws and dressing approach to the integrable models, in "Integrable Hierachies and Modern Physical Theories, Kluwer Academic Publishers, Dordrecht, 2001.

[AvdL] H. Aratyn, J. van de Leur, Solutions of the WDVV Equations and Integrable Hierarchies of KP type, arXiv: hep-th/0104092.

[DJKM] E. Date, M. Jimbo, M. Kashiwara, T. Miwa, Transformation groups for soliton equations III, Operator approach to the Kadomtsev-Petviashvili equation, J. Phys. Soc. Japan 50, no. 11, (1981), 3806-3812.

[Du92a] B. Dubrovin, Integrable systems in topological field theory, Nucl. Phys. B379, 627(1992).

[Du92b] B. Dubrovin, Integrable systems and classification of 2-dimensional topological field theories, Integrable systems (Luminy, 1991), Birkhäuser, Boston, MA, 313-359.

[Du96] B. Dubrovin, Geometry of $2 D$ topological field theories, Integrable systems and quantum groups (Montecatini Terme, 1993), Springer, Berlin, 1996, 120-348.

[DZ98] B . Dubrovin, Y . Zhang, Bihamiltonian hierarchies in 2D topological field theory at one-loop approximation, Comm. Math. Ph. 198, 311(1998).

[HL98] G. F. Helminck and J. W. van de Leur, An analytic description of the vector constrained KP hierarchy, Comm. Math. Phys., 193(1998), no. 3, 627-641.

[HL00] G. F. Helminck, J. W. van de Leur, A geometric construction of the Dual Wavefunction of the n-component KP-hierarchy, Lie Theory and its Applications III, World Scientific, Singapore, 2000, 187-203.

[HP91] G. F. Helminck, G. F. Post, A convergent framework for the multicomponent KP-hierarchy, Trans. Amer. Math. Soc. 324, no. 1, (1991), 187-292.

[HP94] G. F. Helminck, G. F. Post, The geometry of differential difference equations, Indag. Mathem., N. S., 5, no. 4, (1994), 411-438.

[HKM01] L. K. Hoevenaars, P. H. M. Kersten, R. Martini, Generalized WDVV equations for $F_{4}$ pure $N=2$ superYang-Mills theory, Physics Letters B 503(2001), 189-196.

[IY98] K. Ito, S. -K. Yang, WDVV in N=2 SUSY Yang-Mills theory, Physics Letters B 433 (1998), 56-62. 
[Kric92a] I. M. Krichever, The dispersionless Lax equations and topological minimal models, Comm. Math. Phys. 143, no. 2, 415-429.

[Kric92b] I. M. Krichever, The $\tau$-function of the universal Whitham hierarchy, matrix models and topological field theories, Comm. Pure Appl. Math. 47, no. 4, 437-475

[LM99] J. W. van de Leur, R. Martini, The construction of Frobenius manifolds and KP tau-functions, Comm. Math. Phys. 205, no. 3, (1999), 587-616.

[Manin99] Y. Manin, Frobenius manifolds, quantum cohomology, and moduli spaces, American Mathematical Society Colloquium Publications, 47. American Mathematical Society, Providence, RI, 1999.

[MMM97] A. Marshakov, A. Mironov, A. Morozov, WDVV-like equations in $N=2$ SUSY Yang-Mills theory, Phys Letters B 389(1996), 43.

[Mir] A. Mironov, Seiberg-Witten Theories, Integrable models and Perturbative Prepotentials, arXiv:hep-th/0010078.

[vdL] J. van de Leur, Twisted $\mathrm{GL}_{n}$ loop group orbit and solutions of the WDVV equations, Internat. Math. Res. Notices 2001, no. 11, 551-573.

[Pav] O. Pavlyk, Solutions to WDVV from generalized Drinfeld-Sokolov hierarchies, arXiv:math-ph/0003020.

Department of Mathematics, Universiteit Twente, Enschede, The Netherlands

E-mail address: helminck@math.utwente.nl 